\title{
Effectiveness of dry needling in the treatment of neck pain and disability associated with myofascial trigger points
}

\author{
Canan SANAL TOPRAK ${ }^{1}$ (D), Tugba OZSOY UNUBOL ${ }^{2}$ (D) \\ ${ }^{1}$ Department of Physical Medicine and Rehabilitation,School of Medicine, Marmara University, Istanbul, Turkey. \\ ${ }^{2}$ Physical Medicine and Rehabilitation, Sultan Abdulhamid Han Training and Research Hospital, Istanbul, Turkey.
}

Corresponding Author: Canan SANAL TOPRAK

E-mail: canansanal@hotmail.com

Submitted: 21.08.2020 Accepted: 13.11.2020

\begin{abstract}
Objective: The aim of this study is to evaluate the effectiveness of dry needling (DN) with fast-in and fast-out technique for myofascial trigger points (MTrPs) in the upper trapezius muscle associated with neck pain.

Patients and Methods: Patients aged 18-70 years, who have had neck pain at least one active MTrP in the upper trapezius muscle treated with DN, were included in the study. Pain and disability were assessed with Numeric Rating Scale (NRS) and Neck Disability Index (NDI) before treatment (T0), after first session (T1) and after last session (T2). Number of MTrPs where DN was performed and the number of DN sessions were recorded. Patients were evaluated based on the minimal clinically important change (MCIC) scores for NRS and NDI.

Results: A total of 76 patients (Female: 67, Male: 9) were included in the study. Median number of DN sessions was 3 and median of MTrPs that DN performed was 4. Both NRS and NDI showed significant improvement at T1 and T2. More than $90 \%$ of patients had $\mathrm{MCIC}$ at $\mathrm{T} 1$ and $\mathrm{T} 2$.

Conclusion: Dry needling with fast-in and fast-out technique is effective for pain and disability management in patients with neck pain due to MTrPs.

Keywords: Dry needling, Myofascial trigger points, Neck pain, Neck disability
\end{abstract}

\section{INTRODUCTION}

A myofascial trigger point (MTrP), defined as a hyperirritable nodule in a taut band of the muscle, is a neuromuscular dysfunction caused by the unaccustomed overload of the muscles [1]. The compression of the trigger point is painful, provokes the characteristic referred pain and sensation of the patient, and may cause sensory, motor and autonomic symptoms [2]. Repetitive minor microtraumas, acute musculoskeletal injuries, sudden eccentric or concentric contractions, postural and emotional stress, compressive loading and dehydration are thought to play a role in its etiology. Although, the underlying mechanism of the MTrP has not been clearly defined, inappropriate activity of acetylcholine $(\mathrm{ACh})$ at the neuromuscular junction is the most accepted hypothesis [3]. The inhibition of ACh esterase and upregulation of ACh receptors, as well as the excessive release of ACh, are associated with the development of taut bands and trigger points due to a sustained sarcomere contraction which leads to increased metabolism, local ischemia and hypoxia [4]. Consequently, the increased release of algogenic and sensitizing substances from the damaged tissues causes peripheral and central sensitization [5-7]. There are two types of MTrPs: active and latent [8]. While active MTrPs are spontaneously painful, latent MTrPs are painful with palpation and motion but not spontaneously. However, both may result in decreased range of motion, muscle weakness and dysfunction, and reproduce the symptoms of a variety of chronic pain disorders such as tensiontype headache, migraine, temporomandibular joint disorders, mechanical neck pain, shoulder pain, lateral epicondylalgia, low back pain, plantar fasciitis and chronic pelvic pain [2,7].

Dry needling (DN) is a commonly used and increasingly interested non-pharmacological treatment method in which a filiform needle is used to penetrate the MTrP and disrupt the

How to cite this article: Sanal Toprak C, Ozsoy Unubol T. Effectiveness of dry needling in the treatment of neck pain and disability associated with myofascial trigger points. Marmara Med J 2021;34(1):40-44. doi: 10.5472/marumj.866557 
integrity of dysfunctional endplates located near the MTrP [9]. It is considered that DN creates biochemical and neurophysiological changes at and near the MTrP and normalizes the hypersensitive structures $[4,9]$. There are different types of DN: fast-in and fast-out needling such as pistoning (dynamic model), leaving the needle in situ (static model) and rotating the needle several revolutions [6]. There is a debate as to which technique is ideal due to a lack of high-quality studies $[6,10]$. Although, it is conflicting in the literature, some authors suggested that to elicit local twitch response (LTR), an involuntary spinal cord reflex, is important for effectiveness of dry needling [11].

In the literature, there are a growing number of studies which report that $\mathrm{DN}$ of the trapezius muscle has beneficial effects on pain, cervical range of motion, functions and quality of life [12]. However, more research is needed, since the optimal number, duration and intensity of $\mathrm{DN}$ has yet to be determined for the successful treatment.

This study aims to evaluate whether there is a significant change in the pain and neck disability scores of patients with fast-in and fast-out DN technique, and to assess the number of sessions required to achieve the optimal treatment.

\section{PATIENTS and METHODS}

The study protocol was approved by the local ethics committee of Marmara University School of Medicine (approval number 09.617). The study was conducted in accordance with the principles of the Declaration of Helsinki.

\section{Study Design}

Patients who had DN therapy at Marmara University Hospital and Sultan Abdulhamid Han Training and Research Hospital, Department of Physical Medicine and Rehabilitation, between January 2019 and January 2020, were retrospectively scanned from hospital records. Patients aged 18 to 70 years who presented with neck pain, had at least one active MTrP in the upper trapezius muscle and were treated with $\mathrm{DN}$ were included. The exclusion criteria were major trauma documented from the medical history, cervical radiculopathy, pregnancy, inflammatory, hormonal and neurological disorders, and severe psychiatric illness.

Patients who had baseline (T0), after first treatment session (T1) and after last session (T2) data were included in the study. The required data included the following: Numeric Rating Scale (NRS) for pain intensity and Neck Disability Index (NDI) for disability assessment. NRS is an 11-point numeric scale which ranges from 0 (no pain) to 10 (worst pain imaginable). NDI is a 10 -item scale where each item is scored from 0 to 5 , with the total score being the sum of all item scores; higher scores reflect more severe disability. Its validity and reliability has been studied in the Turkish population [13]. NDI was categorized as no disability (0-4), mild (5-14), moderate (15-24), severe (2534 ) and complete (35-50) disability [14]. A minimal clinically important change (MCIC) for NRS and NDI in patients with neck pain has been reported to be 2.5 and 3.5, respectively (15).

\section{Procedure}

Dry needling was performed for each MTrP in the upper trapezius, using a $25 \times 0.25 \mathrm{~mm}$ acupuncture needle with a guided tube, once a week by two physicians with more than 5 years' experience in DN.

The method described by Hong was used. The needle was inserted into the active MTrP. When the first LTR was obtained, multiple rapid insertions of the needle were performed, in and out of the MTrP with fast-in and fast-out technique to get more LTRs $[16,17]$. The procedure was performed while the patients were lying in the prone position. A combination of stretching and strengthening exercises were recommended to all patients.

The number of MTrPs on which DN was performed and the number of $\mathrm{DN}$ sessions were recorded.

\section{Statistical Analysis}

The IBM SPSS Statistics 22 (SPSS IBM, Turkey) program was used for statistical analysis. The normality of the parameters was assessed with the Shapiro-Wilk test. Mean, median, standard deviation, minimum, maximum and frequency were used as the descriptive statistical methods. The Friedman test was used for repeated comparisons and the Wilcoxon signed test was performed with a Bonferroni correction for pairwise comparisons. The Spearman test was performed for correlation analysis. Results were evaluated at a 95\% confidence interval, $\mathrm{p}$ $<0.05$ significance level. After Bonferroni correction $\mathrm{p}<0.017$ was considered statistically significant.

\section{RESULTS}

Overall, 76 patients fulfilling the inclusion criteria (Female: 67, Male: 9), among a total of 104 patients who had trapezius DN applied due to neck pain, were included in the study. The demographic and clinical data are summarized in Table I.

The median number of DN sessions performed was 3, although, in 3 patients only one session of DN was performed because the patients' pain and disability recovered totally. The median number of MTrP that DN was performed on was 4 (Table I).

Table I. Clinical and demographic data of the patients

\begin{tabular}{|c|c|c|}
\hline \multicolumn{2}{|c|}{$\operatorname{Age}_{\text {(vears) }}(\operatorname{mean} \pm \mathrm{SD})(\min -\max )$} & $40.72 \pm 11.66(22-70)$ \\
\hline \multicolumn{2}{|c|}{ Gender (Female/Male) } & $67 / 9$ \\
\hline \multirow[t]{3}{*}{ Comorbidities } & Hypertension (n) & 13 \\
\hline & Diabetes mellitus ( $\mathrm{n}$ ) & 6 \\
\hline & Hypothyroidism (n) & 1 \\
\hline \multicolumn{2}{|c|}{ Symptom duration $_{\text {(months) }}($ median) (min-max) } & $12(2-48)$ \\
\hline \multicolumn{2}{|c|}{ Number of DN sessions (median) (min-max) } & $3(1-10)$ \\
\hline \multicolumn{2}{|c|}{ Number of MTrP (median) (min-max) } & $4(2-6)$ \\
\hline
\end{tabular}

Neck pain and disability scores had significantly reduced at T1 and T2 (Figure 1) (Table II). When MCIC was analysed for NRS 
and NDI, more than $90 \%$ of patients had MCIC at T1 and T2 (Table III).

Table II. Pain and disability outcomes of the patients

\begin{tabular}{|c|c|c|c|}
\hline & & NRS & NDI \\
\hline T0 median (min-max) & & $7(5-10)$ & $18(8-35)$ \\
\hline T1 median (min-max) & & $3(0-7)$ & $8(0-28)$ \\
\hline T2 median (min-max) & & $3(0-8)$ & $4(0-28)$ \\
\hline $\mathrm{p}^{*}$ & & 0.000 & 0.000 \\
\hline $\mathrm{p}^{* *}$ & $\begin{array}{l}\text { T0-T1 } \\
\text { T0-T2 }\end{array}$ & $\begin{array}{l}0.000 \\
0.000\end{array}$ & $\begin{array}{l}0.000 \\
0.000\end{array}$ \\
\hline
\end{tabular}

${ }^{*}$ Friedman test, ${ }^{* *}$ Wilcoxon signed-rank test

NDI: Neck Disability Index, NRS: Numeric Rating Scale, min: minimum, max: maximum

T0: baseline, T1: after first treatment session, T2: after last treatment session

Table III. Clinical important change of pain and disability scores

\begin{tabular}{lcc} 
& T0-T1 $(\mathrm{n}=76)$ & T0-T2 $(\mathrm{n}=73)$ \\
$\mathrm{NRS}_{\text {MCIC }} \mathrm{n}(\%)$ & $69(90.7)$ & $67(91.7)$ \\
\hline $\mathrm{NDI}_{\text {MCIC }} \mathrm{n}(\%)$ & $72(94.7)$ & $71(97.2)$ \\
\hline
\end{tabular}

NDI: Neck Disability Index, NRS: Numeric Rating Scale, MCIC: Minimal Clinical Important Change, T0: baseline, T1: after first treatment session, T2: after last treatment session

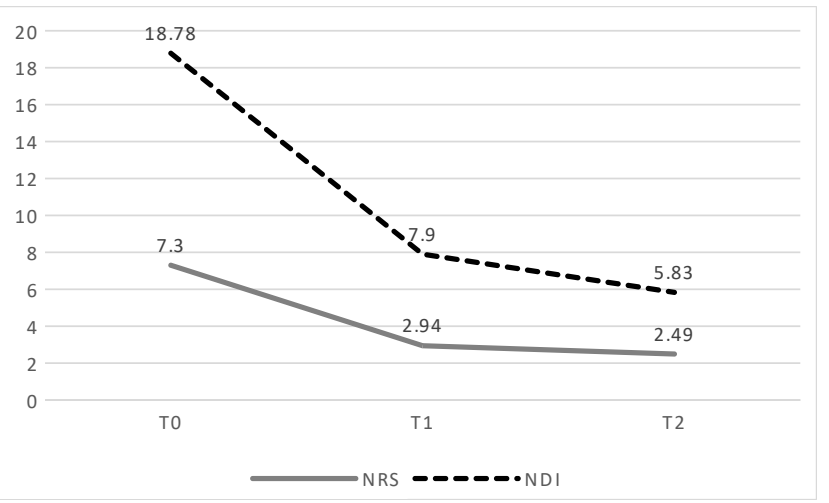

Figure 1. Pain and disability score change over time

There was a moderate positive correlation between symptom duration, number of DN sessions and number of MTrP. There is a weak negative correlation between symptom duration and change of NRS and NDI. A weak positive correlation was detected between the number of DN sessions and change of NDI. In addition, there was a moderate positive correlation between the number of MTrP on which DN was performed and change of NDI (Table IV).
Table IV. Correlation analysis between dry needling parameters and change of pain, disability scores

\begin{tabular}{lcccc}
\hline & & $\begin{array}{c}\text { Symptom } \\
\text { duration }\end{array}$ & $\begin{array}{c}\text { Number of DN } \\
\text { session }\end{array}$ & $\begin{array}{c}\text { Number of } \\
\text { MTrP }\end{array}$ \\
\hline Symptom & $\mathrm{p}$ & - & $\mathbf{0 . 0 0 0}$ & $\mathbf{0 . 0 0 0}$ \\
duration & $\mathrm{r}$ & & $\mathbf{0 . 5 0 3}$ & $\mathbf{0 . 5 1 4}$ \\
\hline Number of & $\mathrm{p}$ & $\mathbf{0 . 0 0 0}$ & - & $\mathbf{0 . 0 0 0}$ \\
DN session & $\mathrm{r}$ & $\mathbf{0 . 5 0 3}$ & & $\mathbf{0 . 4 4 7}$ \\
\hline Number of & $\mathrm{p}$ & $\mathbf{0 . 0 0 0}$ & $\mathbf{0 . 0 0 0}$ & - \\
MTrP & $\mathrm{r}$ & $\mathbf{0 . 5 1 4}$ & $\mathbf{0 . 4 4 7}$ & \\
\hline NRS T0-T1 & $\mathrm{p}$ & $\mathbf{0 . 0 3 5}$ & 0.669 & 0.766 \\
& $\mathrm{r}$ & $\mathbf{- 0 . 2 4 2}$ & -0.050 & -0.035 \\
\hline NRS T0-T2 & $\mathrm{p}$ & $\mathbf{0 . 0 4 6}$ & 0.839 & 0.606 \\
& $\mathrm{r}$ & $\mathbf{- 0 . 2 3 4}$ & 0.024 & -0.061 \\
\hline NDI T0-T1 & $\mathrm{p}$ & 0.282 & 0.113 & $\mathbf{0 . 0 0 0}$ \\
& $\mathrm{r}$ & 0.125 & 0.183 & $\mathbf{0 . 4 8 8}$ \\
\hline NDI T0-T2 & $\mathrm{p}$ & $\mathbf{0 . 0 3 0}$ & $\mathbf{0 . 0 0 2}$ & $\mathbf{0 . 0 0 0}$ \\
& $\mathrm{r}$ & $\mathbf{0 . 2 5 5}$ & $\mathbf{0 . 3 6 5}$ & $\mathbf{0 . 4 6 9}$ \\
\hline
\end{tabular}

Spearman test, DN: Dry Needling, MTrP: Myofascial trigger point, T0: baseline, T1: after first treatment session, T2: after last treatment session

There were no recorded early or delayed procedure-related complications except post needling local soreness, which lasted no longer than 72 hours.

\section{DISCUSSION}

This study revealed that DN of MTrP on the trapezius muscle is an effective method in patients with neck pain. In the past two to three decades, there have been an increasing number of studies in which the beneficial effects of $\mathrm{DN}$ have been demonstrated on neck disability and pain [12,18-23]. However, different types of DN modalities were used in these studies. Therefore, the optimal treatment modalities are unknown and more studies are required in this trending topic to provide more information. In this study, we used fast-in and fast-out technique and aimed to obtain LTRs. Although, there is an expert opinion about this technique which suggests that it is more beneficial than other techniques, evidence regarding the efficiency of fast-in and fast-out technique is limited in the literature [6]. In accordance with previous studies, the current study showed that pain and disability scores of patients were significantly improved after both first and last session with DN using fast-in and fast-out technique $[23,24]$. Additionally, for the interpretation of treatment effects, it is not only important to know whether the results of outcome measurements are significantly changed, but also whether these changes are meaningful for patients $(15,25)$. MCIC is strongly recommended for evaluation of relevant changes in clinical practice and research. In this study, we used the cut-off points of 3.5 for NDI and 2.5 for NRS which were determined by patients and clinicians as MCIC for patients with neck pain in a previous study [15]. Based on these values, more than $90 \%$ of patients had minimal clinical improvement for both outcome measures in this study. 
One of the frequent questions about DN is how many sessions should be applied [6]. In the current study, the number of DN sessions was in line with previous studies, ranging from 1 to 10 with a median of $3[6,23]$. We limited the number of $\mathrm{DN}$ to a maximum of 10 , otherwise the maximum number of DN would have been higher than 10 . We also demonstrated that the number of DN sessions correlated with symptom duration and the number of MTrPs. This also supports the idea that, while subacute MTrPs resolve with less number of DN sessions, chronic MTrPs require more sessions [6]. This result was thought to be related with the central sensitization mechanism of MTrPs. It has been suggested that MTrPs cause a continued peripheral nociceptive afferent bombardment into the dorsal root ganglia, dorsal horn neurons and central nervous system; therefore, the presence of multiple MTrPs, or the presence of MTrPs for a long time, may lead to spinal and supra-spinal sensitization [26], Although, DN is considered to be able to reduce central sensitization by reducing peripheral nociception and dorsal horn neuron activity, and by activating central pain-inhibitory pathways, the treatment of patients with central sensitization is more difficult than those without central sensitization [7]. Short term improvements in pain may occur in patients with central sensitization; however, treatment modalities which have beneficial effects on central sensitization should be considered, to improve patients in the long term. In patients who have beneficial effects on pain with $\mathrm{DN}$ but require a multiple number of $\mathrm{DN}$, as in the current study, clinicians should be aware that the effectiveness of DN may be short due to central sensitization.

\section{Limitations}

First of all, a control group, treated with other DN techniques or other treatment methods such as physical therapy, ischemic compression and manual therapy, could not be included in this study due to its retrospective design. In addition, we were not able to supervise whether patients performed exercises regularly, as recommended by physicians. Secondly, we did not evaluate the long-term results of $\mathrm{DN}$ which might have been essential, especially for patients where DN was performed 10 times. We consider that these patients might have had central sensitization and the duration of DN efficacy in these patients would therefore have been shorter than others.

\section{Conclusion}

This study demonstrated that DN with fast-in and fast-out technique should be considered in the management of patients with neck pain due to MTrPs. It has beneficial effects on both pain and disability. More randomized control studies are needed to compare $\mathrm{DN}$ techniques and modalities and also to evaluate related factors with the efficacy of DN.

\section{Compliance with Ethical Standards}

Ethical Approval: All procedures performed in accordance with the ethical standards of the institutional and/or national research committee and with the 1964 Helsinki Declaration and its later amendments or comparable ethical standards. The study was approved by the Ethics Committee of Marmara University School of Medicine (approval number: 09.617).

Financial Support: The authors have no relevant financial information to disclose.

Conflict of Interest: The authors have no conflict of interest to declare.

Author Contributions: The manuscript has two authors and each author is responsible for the content and writing of the paper.

\section{REFERENCES}

[1] Navarro-Santana MJ, Sanchez-Infante J, Fernández-de-LasPeñas C, et al. Effectiveness of dry needling for myofascial trigger points associated with neck pain symptoms: An updated systematic review and meta-analysis. J Clin Med 2020;9: 3300. doi: 10.3390/jcm9103300

[2] Shah JP, Thaker N, Heimur J, et al. Myofascial trigger points then and now: a historical and scientific perspective. $P M \& R$ 2015:7: 746-61. doi: 10.1016/j.pmrj.2015.01.024

[3] Climent JM, Kuan T-S, Fenollosa P, et al. Botulinum toxin for the treatment of myofascial pain syndromes involving the neck and back: a review from a clinical perspective. Evid Based Complement Alternat Med 2013:2013. doi: 10.1155/2013/381459

[4] Cagnie B, Dewitte V, Barbe T, et al. Physiologic effects of dry needling. Curr Pain Headache Rep 2013;17: 348. doi: 10.1007/ s1 1916.013.0348-5

[5] Shah JP, Danoff JV, Desai MJ, et al. Biochemicals associated with pain and inflammation are elevated in sites near to and remote from active myofascial trigger points. Arch Phys Med Rehabil 2008;89: 16-23. doi: 10.1016/j.apmr.2007.10.018

[6] Unverzagt C, Berglund K, Thomas J. Dry needling for myofascial trigger point pain: a clinical commentary. Int $\mathrm{J}$ Sports Phys Ther 2015;10: 402-18.

[7] Fernández-de-Las-Peñas C, Nijs J. Trigger point dry needling for the treatment of myofascial pain syndrome: current perspectives within a pain neuroscience paradigm. J Pain Res 2019;12: 1899-1911. doi: 10.2147/JPR.S154728

[8] Pecos-Martín D, Montañez-Aguilera FJ, Gallego-Izquierdo T, et al. Effectiveness of dry needling on the lower trapezius in patients with mechanical neck pain: a randomized controlled trial. Arch Phys Med Rehabil 2015;96: 775-81. doi: 10.1016/j. apmr.2014.12.016

[9] Dommerholt J. Dry needling-peripheral and central considerations. J Man Manip Ther 2011;19: 223-7. doi: 10.1179/106698111X131.297.29552065

[10] Dunning J, Butts R, Mourad F, et al. Dry needling: a literature review with implications for clinical practice guidelines. Phys Ther Rev 2014;19: 252-65. doi: 10.1179/108331913X138.442.45102034

[11] Tsai C-T, Hsieh L-F, Kuan T-S, et al. Remote effects of dry needling on the irritability of the myofascial trigger point in 
the upper trapezius muscle. Am J Phys Med Rehabil 2010;89: 133-40. doi: 10.1097/PHM.0b013e3181a5b1bc

[12] Liu L, Huang Q-M, Liu Q-G, et al. Effectiveness of dry needling for myofascial trigger points associated with neck and shoulder pain: a systematic review and meta-analysis. Arch Phys Med Rehabil 2015;96: 944-55. doi: 10.1016/j. apmr.2014.12.015

[13] Aslan E, Karaduman A, Yakut Y, et al. The cultural adaptation, reliability and validity of neck disability index in patients with neck pain: a Turkish version study. Spine 2008;33: E362-E365. doi: 10.1097/BRS.0b013e31817144e1

[14] Cleland JA, Fritz JM, Whitman JM, et al. The reliability and construct validity of the Neck Disability Index and patient specific functional scale in patients with cervical radiculopathy. Spine 2006;31: 598-602. doi: 10.1097/01. brs.000.020.1241.90914.22

[15] Pool JJ, Ostelo RW, Hoving JL, et al. Minimal clinically important change of the Neck Disability Index and the Numerical Rating Scale for patients with neck pain. Spine 2007;32: 3047-51.doi: 10.1097/BRS.0b013e31815cf75b

[16] Simons DG, Travell J, Simons LS. Myofascial pain and dysfunction: the trigger point manual. Volume 1. Baltimore: Williams and Wilkins, 1999.

[17] Hong C-Z. Considerations and recommendations regarding myofascial trigger point injection. J Musculoskelet Pain 1994;2: 29-59.doi: 10.1300/J094v02n01_03

[18] Tekin L, Akarsu S, Durmuş O, et al. The effect of dry needling in the treatment of myofascial pain syndrome: a randomized double-blinded placebo-controlled trial. Clin Rheumatol 2013;32: 309-15.doi: 10.1007/s10067.012.2112-3

[19] Onat SS, Polat CS, Bicer S, et al. Effect of dry needling injection and kinesiotaping on pain and quality of life in patients with mechanical neck pain. Pain physician 2019;22: 583-9.
[20] Cagnie B, Castelein B, Pollie F, et al. Evidence for the use of ischemic compression and dry needling in the management of trigger points of the upper trapezius in patients with neck pain: a systematic review. Am J Phys Med Rehabil 2015;94: 573-83.doi: 10.1097/PHM.000.000.0000000266

[21] Cerezo-Téllez E, Torres-Lacomba M, Fuentes-Gallardo I, et al. Effectiveness of dry needling for chronic nonspecific neck pain: a randomized, single-blinded, clinical trial. Pain 2016;157: 1905-17.doi: 10.1097/j.pain.000.000.0000000591

[22] Gallego-Sendarrubias GM, Rodríguez-Sanz D, Calvo-Lobo C, et al. Efficacy of dry needling as an adjunct to manual therapy for patients with chronic mechanical neck pain: a randomised clinical trial. Acupunct Med 2020; acupmed-2018-011682. doi: 10.1097/AJP.000.000.0000000634

[23] Ziaeifar M, Arab AM, Mosallanezhad Z, et al. Dry needling versus trigger point compression of the upper trapezius: a randomized clinical trial with two-week and threemonth follow-up. J Man Manip Ther 2019;27: 152-161. doi: 10.1080/10669.817.2018.1530421

[24] Kietrys DM, Palombaro KM, Azzaretto E, et al. Effectiveness of dry needling for upper-quarter myofascial pain: a systematic review and meta-analysis. J Orthop Sports Phys Ther 2013;43: 620-634. doi: 10.2519/jospt.2013.4668

[25] Nunes AMP, Moita JPAM. Effectiveness of physical and rehabilitation techniques in reducing pain in chronic trapezius myalgia: A systematic review and meta-analysis. Int J Osteopath Med 2015;18: 189-206.doi: 10.1016/j. ijosm.2015.03.004

[26] Fernández-de-las-Peñas C, Dommerholt J. Myofascial trigger points: peripheral or central phenomenon? Curr Rheumatol Rep 2014;16: 395.doi: 10.1007/s11926.013.0395-2 